\title{
Synchronization of Neural Activity across Cortical Areas Correlates with Conscious Perception
}

\author{
Lucia Melloni, ${ }^{1 \star}$ Carlos Molina, ${ }^{1,2}$ Marcela Pena, ${ }^{1,3}$ David Torres, ${ }^{1}$ Wolf Singer, ${ }^{4,5}$ and Eugenio Rodriguez ${ }^{1,4,5 *}$ \\ ${ }^{1}$ Laboratorio de Neurociencias, Escuela de Psicología, Pontificia Universidad Católica de Chile, Vicuña Mackenna 4860, San Joaquin, 8940000 Santiago, \\ Chile, ${ }^{2}$ Radboud University Nijmegen, 6525 EK Nijmegen, The Netherlands, ${ }^{3}$ International School for Advanced Studies, Cognitive Neuroscience Sector, \\ 34014 Trieste, Italy, ${ }^{4}$ Department of Neurophysiology, Max Planck Institute for Brain Research, 60528 Frankfurt am Main, Germany, and ${ }^{5}$ Frankfurt \\ Institute for Advanced Studies, Johann Wolfgang Goethe University, 60438 Frankfurt am Main, Germany
}

Subliminal stimuli can be deeply processed and activate similar brain areas as consciously perceived stimuli. This raises the question which signatures of neural activity critically differentiate conscious from unconscious processing. Transient synchronization of neural activity has been proposed as a neural correlate of conscious perception. Here we test this proposal by comparing the electrophysiological responses related to the processing of visible and invisible words in a delayed matching to sample task. Both perceived and nonperceived words caused a similar increase of local (gamma) oscillations in the EEG, but only perceived words induced a transient long-distance synchronization of gamma oscillations across widely separated regions of the brain. After this transient period of temporal coordination, the electrographic signatures of conscious and unconscious processes continue to diverge. Only words reported as perceived induced (1) enhanced theta oscillations over frontal regions during the maintenance interval, (2) an increase of the P300 component of the eventrelated potential, and (3) an increase in power and phase synchrony of gamma oscillations before the anticipated presentation of the test word. We propose that the critical process mediating the access to conscious perception is the early transient global increase of phase synchrony of oscillatory activity in the gamma frequency range.

Key words: visual awareness; electroencephalography; event-related potentials; gamma-band oscillations; long-range coordination; oscillatory neural synchrony

\section{Introduction}

Stimuli that are masked and presented so briefly that they cannot be reported can still be highly processed and even activate motor responses (Marcel, 1983; Dehaene et al., 1998, 2001, 2004; Naccache et al., 2005). Evidence from patients suffering from blindsight (Goebel et al., 2001), hemineglect (Vuilleumier et al., 2002; Cappelletti and Cipolotti, 2006), or prosopagnosia (Renault et al., 1989) supports the notion that unconsciously processed stimuli activate high-level cortical areas. This implies that complex cognition can proceed in the absence of consciousness, raising the question how the neuronal substrates of conscious and nonconscious processes differ. Several studies have suggested that conscious perception associates with enhanced sensory responses (Grill-Spector et al., 2000; Bar et al., 2001) and with activation of areas higher in the processing hierarchy (Beck et al., 2001; Dehaene et al., 2001; Kleinschmidt et al., 2002; Marois et al., 2004; Carmel et al., 2005). However, other studies suggest that this may

\footnotetext{
Received 0ct. 25, 2006; revised Jan. 16, 2007; accepted Feb. 5, 2007.

L.M. was supported by the Comisión Nacional de Investigación Científica y Tecnológica (Chile) and Deutscher Akademischer Austausch Dienst (Germany). E.R. was supported by Max Planck Gesellchaft, Volkswagen Stiftung, and the Frankfurt Institute for Advanced Studies.

*L.M. and E.R. contributed equally to this work.

Correspondence should be addressed to Dr. Lucia Melloni, Brain Imaging Center and Cognitive Neurology Unit, Johann Wolfgang-Goethe Universität, Schleusenweg 2-16, 60528 Frankfurt am Main, Germany. E-mail: melloni@mpih-frankfurt.mpg.de.

DOI:10.1523/JNEUROSCI.4623-06.2007

Copyright $\odot 2007$ Society for Neuroscience $\quad 0270-6474 / 07 / 272858-08 \$ 15.00 / 0$
}

not be a sufficient condition, because invisible stimuli activate similar structures as visible stimuli (Moutoussis and Zeki, 2002, 2006).

Alternatively, it has been proposed that conscious perception depends on the transient synchronization of widely distributed neural assemblies (Engel et al., 1999; Engel and Singer, 2001; Thompson and Varela, 2001; Singer, 2002). The neural signature of unconscious perception would be local coordination of neural activity and propagation along sensory processing pathways, whereas conscious perception would require global coordination of widely distributed neural activity by long-distance synchronization (Dehaene et al., 2006). Indeed, $\beta$ and gamma frequency band phase synchrony is enhanced for consciously perceived stimuli (Meador et al., 2002; Gross et al., 2004; Nakatani et al., 2005; Palva et al., 2005) and correlates with conscious perception in binocular rivalry (Fries et al., 1997, 2002; Srinivasan et al., 1999; Doesburg et al., 2005). However, these studies have investigated synchronization in spatially restricted neural assemblies, or without distinguishing between local and global coordination. The purpose of this study was to disentangle the role of local processing and global coordination in conscious and unconscious perception and to determine the nature and the time course of electrophysiological events that discriminate between them.

We recorded electroencephalographic (EEG) signals in subjects engaged in a delayed matching to sample task. The visibility 
of the target stimulus was manipulated such that the word was either consciously perceived or remained invisible but was still processed. As indicators of local and global processing (Varela et al., 2001), we measured over a wide frequency range (1) timeresolved power changes of local signals and (2) phase synchronization across recording sites. We found that visible and invisible conditions differed with respect to large-scale synchronization but not local neural processing. In addition, only consciously perceived stimuli gave rise to a cascade of electrographic events that have been proposed to be associated with perception stabilization, maintenance in working memory, and generation of expectancies. We propose that the transient large-scale synchronization is the critical event that triggers these subsequent processes by enhancing the saliency of the activation patterns sufficiently to permit access to consciousness.

\section{Materials and Methods}

\section{Experiment 1}

Subjects. Fifteen normal healthy subjects gave written informed consent to participate in the study (seven males; mean age, 20.6 years). All were native Spanish speakers, right handed, had normal or corrected-tonormal vision, and were naive to the purpose of the experiment.

Stimuli and task. Participants were engaged in a two-alternative, forced-choice-delayed matching to sample task (320 trials), in which the sample stimuli could be either visible or invisible. The subjects' task was to determine as accurately as possible whether a first briefly presented 33 ms "sample word" was the same or different from a second "test word" presented 533 ms later (see Fig. 1a). In each trial, participants responded by pressing one of two different buttons mounted on a response pad, with their right or left index fingers. In seven participants, the same response was at the right button, whereas in eight participants, it was at the left button. The first word was preceded and followed by masking stimuli (67 ms each), which changed in luminance between trials rendering the sample word visible or invisible (experimental conditions). To assess the responses to the masking stream itself, sample words were replaced by a blank screen, while the masking stream was kept constant (control conditions). To render control trials perceptually similar to experimental trials, another word, the "control word," was presented after the mask stream and before test word presentation (see Fig. 1a). This design enabled us to isolate the response to the sample word by subtracting the response elicited by the masking stimuli.

The stimulus set consisted of 40 disyllabic Spanish words. Syllables were consonant-vowel. All words were nouns matched in frequency of usage (S. Sadowsky and R. Martínez, unpublished observation) and presented in Howard light font. The geometric masks were presented in white or gray color (luminance change) on a black background, rendering the sample word visible or invisible. The luminance values used in this experiment were set in a previous control experiment and were kept constant throughout the subjects. Masks were constructed by mixing a number of squares and diamonds drawn with identical line thickness as the font used for the sample words. All stimuli were presented on a computer screen in enhanced graphic adapter mode $(150 \mathrm{~Hz}$ refresh rate), located in the central area of the screen, and subtended $2.5 \times 1^{\circ}$ of visual angle. Each participant ran a training block of 20 trials. Experimental and control trials were pseudo-randomized within four blocks of 80 trials each, interrupted with a variable rest pause. All conditions were equally presented within a block. The intertrial interval had a random length between 1000 and $1500 \mathrm{~ms}$. The experiment was performed in a silent, dimly lit, and electrically shielded room. Special consideration was given to the potential contamination of the EEG signal by the $50 \mathrm{~Hz} \mathrm{AC}$ component, because the gamma band response includes this frequency. This nonbiological artifact was reduced by recording inside a completely hermetic Faraday cage. All of the electrical equipment was outside of the recording room with the only exception of the monitor, which was electrically shielded.

\section{Experiment 2}

Twenty subjects took part in an unconscious semantic priming experiment (11 males; mean age, 23.8 years), with the same word set and masks as used in the previous experiment. All subjects gave written informed consent, were native Spanish speakers, right handed, had normal or corrected-to normal vision, and were naive of the purpose of the experiment. Participants performed a simple semantic classification task by clicking with the left or right index finger (response pattern was reversed for half of the subjects) to indicate whether the target word was natural or manmade, respectively. Unknown to them, another word, which could be either semantically congruent (e.g., pair key-house) or incongruent (e.g., pair key-dog) with the target word was presented for $33 \mathrm{~ms}$ before the target and surrounded by forward and backward masks that rendered it invisible. The duration of the prime and mask were the same as in the previous experiment (mask - prime - mask $=67-33-67 \mathrm{~ms}$, respectively). The target word was presented for $300 \mathrm{~ms}$, and the third mask remained on the screen until subjects responded. The prime-target stimulus onset asynchrony was $100 \mathrm{~ms}$ (33 ms prime $+67 \mathrm{~ms}$ forward mask).

\section{Electrophysiological recording and analysis}

EEG activity was recorded from a 64-electrode geodesic sensor net referenced to the vertex. The electroencephalogram was digitized at $1000 \mathrm{~Hz}$, and the initial bandpass recording filter was set at $0.01-100 \mathrm{~Hz}$.

For the event-related potential (ERP) analysis, the continuous EEG signal was bandpass filtered $(0.5-20 \mathrm{~Hz})$ with a finite impulse response (FIR) filter (Kaiser type), which has a linear phase response [passband gain, 99\% (50-99.9\%; - $0.1 \mathrm{~dB})$; stopband gain, 1\% (1-49.9\%; -40.0 $\mathrm{dB})$; Rollof, $2 \mathrm{~Hz}(0.3-10 \mathrm{~Hz})]$. Then, the filtered signal was segmented in a series of 1100-ms-long epochs. Each epoch started $100 \mathrm{~ms}$ before the onset of the first mask. Trials that contained voltage fluctuations exceeding $\pm 200 \mu \mathrm{V}$, transients exceeding $\pm 100 \mu \mathrm{V}$, or electro-oculogram activity exceeding $\pm 70 \mu \mathrm{V}$ were rejected. Artifact free trials were averaged in synchrony with first mask presentation, digitally transformed to an average reference, and baseline corrected over a $100 \mathrm{~ms}$ window. The EEGLAB Matlab toolbox was used for visualization and topographic plots (Delorme and Makeig, 2004).

For the analysis of time-frequency distributions and phase synchrony, a filter was designed that eliminated only the continuous $50 \mathrm{~Hz}(\mathrm{AC})$ component but kept the biological $50 \mathrm{~Hz}$ signal. The amplitude and phase of the AC signal was estimated and subtracted from the original signal. This eliminated selectively the periodic part of the $50 \mathrm{~Hz}$ component (line frequency). Then, a FIR (350 order Hanning window) bandpass filter $(10-100 \mathrm{~Hz})$ was applied, and the filtered signal was analyzed with a sliding-window fast Fourier transform (window length, $128 \mathrm{~ms}$; step, $10 \mathrm{~ms}$; window overlap, 90\%). For every time window and frequency bin, amplitude and phase were computed as follows: signal windows (128 points) were zero-padded to complete 1024 points and fast Fourier transformed to get an interpolated frequency resolution of $\sim 1$ $\mathrm{Hz}$ per frequency bin. Instantaneous amplitude was then computed by taking the real and imaginary Fourier coefficients $\left(C(f, t)_{\mathrm{r}}\right.$ and $\left.C(f, t)_{\mathrm{i}}\right)$, squaring and adding them, and taking the square root (sqrt) (i.e., for a given time window $t$ and frequency bin $f$ ), as follows:

$$
\operatorname{Amp}(f, t)=\operatorname{sqrt}\left(C(f, t)_{\mathrm{r}}^{2}+C(f, t)_{\mathrm{i}}^{2}\right) .
$$

This amplitude corresponds to the length of the vector specified by the real and imaginary Fourier coefficient computed by Pythagora's law and is equivalent to the magnitude of the observed oscillation at a given time and frequency point.

During the same time window and frequency bin, phase was computed as the arc tangent (arctg) of the imaginary Fourier coefficient divided by the real one, as follows:

$$
\operatorname{Phi}(f, t)=\operatorname{arctg}\left(C(f, t)_{\mathrm{i}} / C(f, t)_{\mathrm{r}}\right) .
$$

Phi is thus the angle of the vector specified by the real and imaginary Fourier coefficient. For a given time and frequency point, it corresponds to a position inside the oscillation cycle (peak, valley, rising, or falling slope).

These amplitude and phase values are evaluated in the $10-100 \mathrm{~Hz}$ frequency range and in the -500 to $+1000 \mathrm{~ms}$ period relative to first 
a)

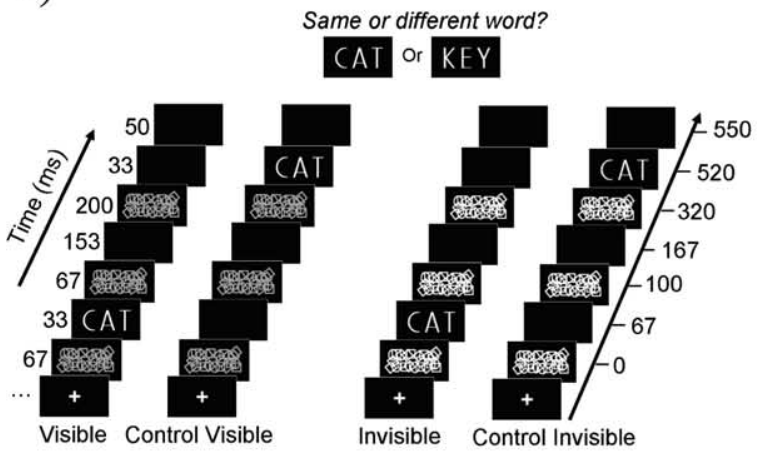

b)

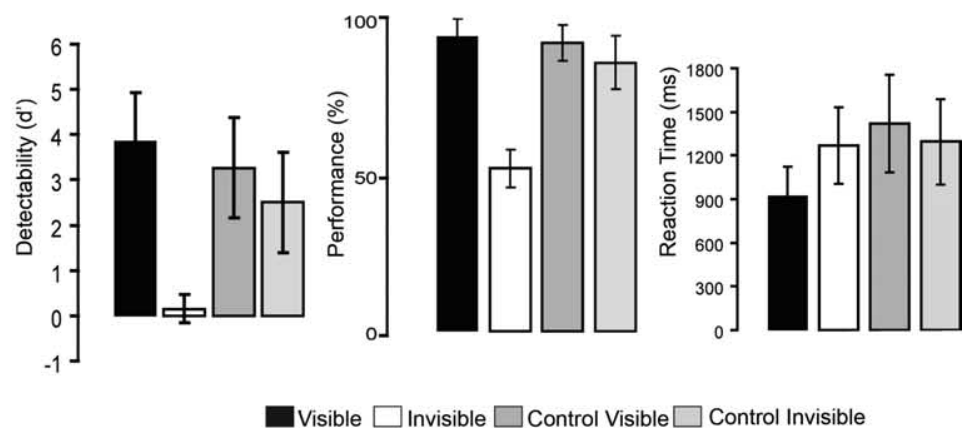

Figure 1. Design and behavioral results of experiment. $\boldsymbol{a}$, Stimulus sequence. The task was to compare a briefly presented word (sample word) with a subsequent word (test word). The sample word visibility was controlled by changing the luminance of the masks. Control conditions were created to assess the brain response to the mask stream. The left timeline shows the duration of each stimulus. The right timeline shows the cumulative time. $\boldsymbol{b}$, Behavioral performance. The left plot shows stimulus detectability for all conditions, expressed as detectability index ( $\left.d^{\prime}\right)$, and the middle plot is the success rate. The right plot shows the reaction time for all conditions. Plots indicate mean performance \pm 1 SD.

mask presentation. Phase information was then used to compute a timevarying phase-locking value (PLV), an index of neural synchrony (Lachaux et al., 1999; Rodriguez et al., 1999). In brief, the method involves computing the phase difference in a time window for an electrode pair and assessing the stability of such phase difference through all trials. If $\phi_{\mathrm{i}}$ and $\phi_{\mathrm{j}}$ are unitary vectors representing the phase of signals in electrodes $\mathbf{i}$ and $\mathbf{j}$, then the phase difference between such electrodes is a unitary vector obtained by multiplying the first vector by the complex conjugate (conj) of the second:

$$
\phi_{\mathrm{ij}}=\phi_{\mathrm{i}} \operatorname{conj}\left(\phi_{\mathbf{j}}\right) .
$$

The PLV is thus the length of the vector resulting from the vector sum of difference vectors through the trials:

$$
\operatorname{PLVij}=\operatorname{abs}\left(1 / N \times \sum \phi_{\mathbf{i j}}\right),
$$

with the sum operating throughout all of the trials and where $N$ is the number of trials. The PLV index ranges from 0 to 1 , with value 1 indicating perfect synchronization (phase difference is perfectly constant throughout the trials) and value 0 representing total absence of synchrony (phase differences are random). Time-frequency charts of both phase synchrony and spectral power were normalized to a baseline of 500 $\mathrm{ms}$ preceding the first mask onset. The normalization involves subtracting the baseline average and dividing by the baseline SD on a frequencyby-frequency basis in the following manner: $S$ is a signal, $\mu$ is the average of the signal during the baseline period, and $\sigma$ is the SD of the same baseline period. Then, the normalized signal is given by the following:

$$
S_{\mathrm{N}}=(S-\mu) / \sigma \text {. }
$$

In another analysis aiming to analyze the theta activity associated with the retention interval (from sample-word offset to test-word onset), a fast Fourier transform was computed over a $467 \mathrm{~ms}$ window, on the raw unfiltered EEG signal. Afterward, the Fourier coefficients corresponding to the theta frequency that showed significantly $(p<0.05)$ higher amplitude $(5-6 \mathrm{~Hz})$ were added over trials and subjects to generate a topographical map of theta activity.

\section{Statistical analysis}

Because we were interested in long-range coordination of neural activity, we included all electrodes in the calculation to produce a global index of synchronization across a large frequency range. Behavioral and EEG data were analyzed with two-tailed, within-subject ANOVA. The $\alpha$ level was set at 0.05 for all tests.

The statistical analyses of the time-frequency distributions and phase synchrony were all performed on time-frequency charts resulting from averaging the electrophysiological responses of all sensors during the entire segment ( -500 to $1000 \mathrm{~ms}$ after sample-word onset). This resulted in a grand average time-frequency and phase-synchrony chart per experimental condition per subject. Then, those charts were grouped by condition and analyzed by means of a permutation test in search of timefrequency windows showing significant effects (Burgess and Gruzelier, 1999). Subsequently, those significant time-frequency windows were analyzed with a two-tailed, within-subject ANOVA. The $\alpha$ level was set at 0.05 for all tests.

In the permutation test, the time-frequency charts belonging to different conditions are mixed to compute a random distribution. This is then used to evaluate the statistical significance of the results. The permutation test assumes that the "real" differences between conditions should exceed the random differences. The permutation test has advantages over the Bonferroni correction for multiple comparisons, because the Bonferroni correction assumes that all measures are independent, an assumption that is too strong and weakens the power of the statistical test. In contrast, the permutation test considers the true dependency among all of the measures.

For the topographical analysis of phase synchrony, we controlled for the statistical effects of multiple comparisons by choosing a very conservative significance threshold $(p<0.00005)$. This threshold was set as a function of the distribution of synchrony values during the baseline. The threshold was chosen to give a probability smaller than 0.00005 . This threshold was computed by counting the number of cases larger than the threshold divided by the total number of cases. This fraction should give a value smaller than 0.00005 . By choosing this significance level, one line per analysis window could be explained, by chance, given the fact that there were 64 electrodes with 2016 possible combinations $(64 \times 63 / 2=$ 2016).

\section{Results}

\section{Behavioral measures of visibility}

Behavioral results showed that, in the experimental condition in which sample words could be perceived, $94.5 \%$ of the words were classified correctly $\left(F_{(1,14)}=904.5 ; p<0.001\right)$, the mean $d^{\prime}$ being 3.85 , which implies a highly significant difference from chance $\left(F_{(1,14)}=192.2 ; p<0.001\right)$. Conversely, in the experimental condition that rendered sample words invisible, performance dropped to chance level $\left(52.2 \% ; F_{(1,14)}=2.097 ; p=0.170\right)$, and the mean $d^{\prime}$ value did not differ from zero $\left(d^{\prime}=0.16 ; F_{(1,14)}=\right.$ 2.598; $p=0.129$ ) (Fig. 1b). In control conditions, the success rates were 92.50 and $86.17 \%$, and the respective $d^{\prime}$ values were 3.27 and 2.50, indicating that participants were able to detect the control words.

Reaction times were significantly shorter in the visible com- 


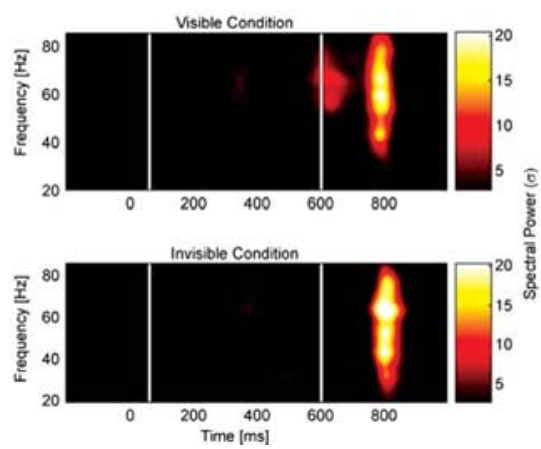

b)
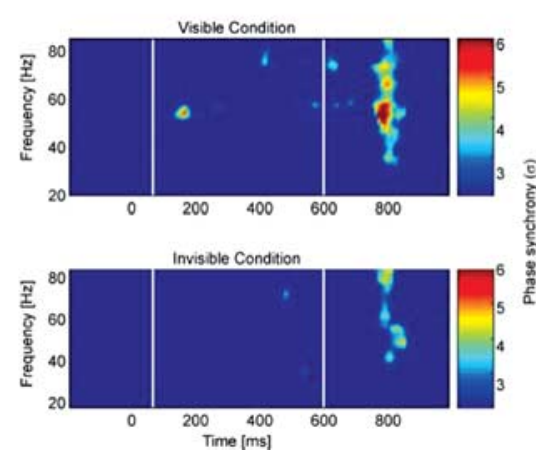

Figure 2. Spectral power and phase synchrony to visible and invisible words. The visible condition (visible - control_visible) and invisible condition (invisible - control_invisible) are shown. The time-frequency plot shows the grand average of all electrodes. The phase-synchrony plot shows the grand average for all of the electrode pairs. The color scale indicates amplitude in SD, calculated over a $500 \mathrm{~ms}$ baseline. Zero corresponds to first mask onset. Vertical lines indicate sample- and test-word presentation. $\boldsymbol{a}$, Time - frequency plot. Two increments of gamma-power emission are visible. The first is only present in the visible condition, and the second is present in both conditions. $\boldsymbol{b}$, Phase synchrony. There are three statistically significant bursts of synchronous activity. The first and second peaks occur only in the visible condition. No significant differences were found for the last peak.

pared with the invisible condition (923 and 1418 ms, respectively; $\left.F_{(1,14)}=76.139 ; p=0.000018\right)$, despite the fact that speed of response was not stressed in the task. Reaction times between the two control conditions did not differ $\left(1269\right.$ and $1296 \mathrm{~ms} ; F_{(1,14)}=$ $0.361 ; p=0.557)$.

To determine to which extent the invisible words in the experimental condition were still processed, we assessed the depth of processing of the invisible words in an unconscious priming task (Marcel, 1983), using the same stream of stimuli (mask-sample word-mask-target word), and determined priming effects with a reaction time task. This control experiment revealed strong priming effects of the invisible word. That is, subjects responded significantly faster in the congruent condition (prime and target word belong to the same semantic category) compared with the incongruent condition (prime and target word belong to different semantic categories) (effect size was $15 \mathrm{~ms}$ difference between congruent and incongruent trials; $n=20$ subjects; $F_{(1,19)}=8.981$; $p=0.007)$. Therefore, masked words, although invisible, were still processed.

\section{Activity patterns related to perceptual awareness}

The analyses described below have all been performed after eliminating effects caused by differences in mask luminance. The EEG traces obtained after presentation of the high and low luminance mask alone (control condition) were subtracted from the traces of the corresponding experimental condition for an analysis window extending from $500 \mathrm{~ms}$ before to $1000 \mathrm{~ms}$ after presentation of the first mask. The subtracted conditions are referred here as "visible" and "invisible," respectively.

The first significant difference between visible and invisible words was observed from 80 to $130 \mathrm{~ms}$ after sample-word presentation. During this period, the mean phase synchrony at $50-57 \mathrm{~Hz}$ over all electrode pairs was significantly higher for the visible than the invisible condition $\left(F_{(1,14)}=5.041 ; p=0.044\right)$ (Fig. $2 b$ ). During the same interval, neither the mean amplitude of the gamma oscillations $\left(50-57 \mathrm{~Hz} ; F_{(1,14)}=0.616 ; p=0.448\right)$ nor the mean ERPs calculated over all as well as over occipital electrodes differed between conditions (ERP effect over all electrodes: $F_{(1,14)}=0.005, p=0.774,0.0245 \mu \mathrm{V}$ difference; ERP effect over occipital electrodes: $F_{(1,14)}=1.036, p=0.329,0.3350$ $\mu \mathrm{V}$ difference) (Figs. 2a, 3).
To obtain more detailed information about activation patterns related to the visible and invisible conditions, regional distributions of gamma spectral power and phase synchrony were computed for the interval from $260 \mathrm{~ms}$ before to $330 \mathrm{~ms}$ after onset of the sample word (frequency, 50-57 Hz; $150 \mathrm{~ms}$ sliding window). Although the patterns of gamma activity were spatially homogeneous and similar for the two conditions, the patterns of phase synchrony were markedly different (Fig. 4). In the invisible condition, only few electrode pairs exhibited significant phase synchronization. In the visible condition, in contrast, numerous electrode pairs, both within and across hemispheres, showed significant phase-locking between occipital, parietal, and frontal sites in the time window $40-180 \mathrm{~ms}$ after sample-word presentation. In the window between 180 and $330 \mathrm{~ms}$, the pattern of phase synchrony became lateralized over the left hemisphere and restricted to occipito-parietal electrodes, which agrees with the fact that the task involves language processing.

The finding that phase-locking in the visible condition increases without a concomitant increase of gamma power, even when the comparison was restricted to individual electrodes, suggests that the main difference between visible and invisible conditions, in this early period, is the coherence of activity among cortical regions rather than the amount of local neural synchronization.

\section{Electrophysiological signatures of further processing of visible stimuli}

A second significant difference between perceptual conditions was apparent in the ERP from 130 to $430 \mathrm{~ms}$ after sample-word presentation. In this interval, the mean amplitude of a frontocentral positivity, peaking at $240 \mathrm{~ms}$ after sample-word presentation and resembling P3a, was higher in the visible than in the invisible condition $\left(F_{(1,14)}=37.766 ; p=0.00002 ; 2.3394 \mu \mathrm{V}\right.$ difference). Interestingly, the amplitude of the P3-like component starts to diverge at a point in time when the differences in phase synchrony have vanished. The P3-like component has been related to the updating of contents kept in working memory (Donchin, 1981). In addition, the mean amplitude of theta activity $(5-6 \mathrm{~Hz})$ increased significantly over frontal electrodes in the visible condition during the interval in which the sample word had to be kept in memory (time interval, 67-520 ms) (see supplemental Fig. 1, available at www.jneurosci.org as supplemental material). Frontal theta oscillations have been assigned a role in the maintenance of short-term memories (Jensen and Tesche, 2002; Schack et al., 2005). Although it would have been interesting to see whether the theta enhancement started only after the episode of gamma phase-locking, the necessity to use long windows for the assessment of the low-frequency oscillations precluded determination of the precise onset of enhanced theta.

A third and more delayed difference between the visible and invisible conditions was observed immediately after test-word presentation. Here, both phase synchrony and gamma power were significantly higher during the visible than the invisible conditions. The increase in synchrony, averaged over all electrodes, occurred in the high gamma range $(67-80 \mathrm{~Hz})$ and during the 
a)

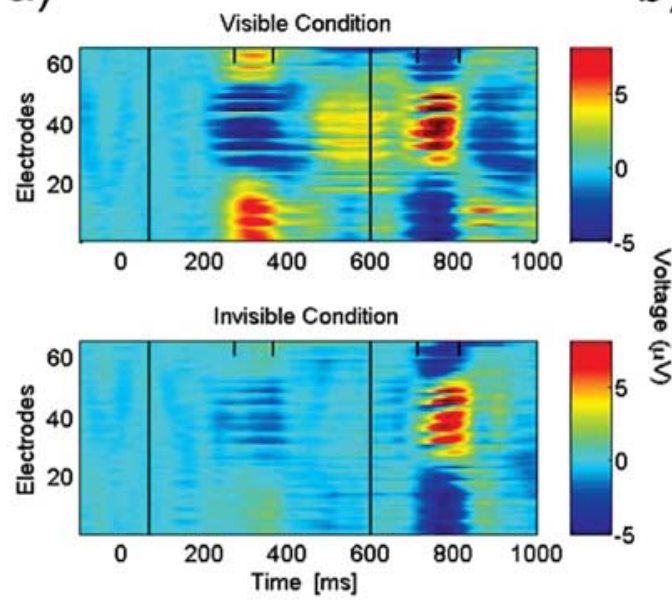

b)

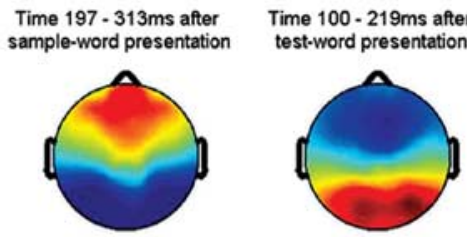

Visible Condition
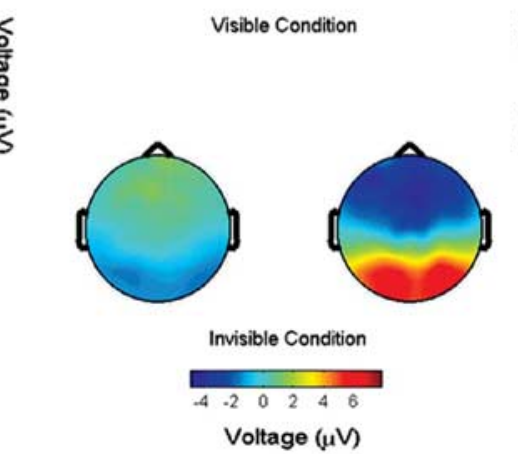

c)

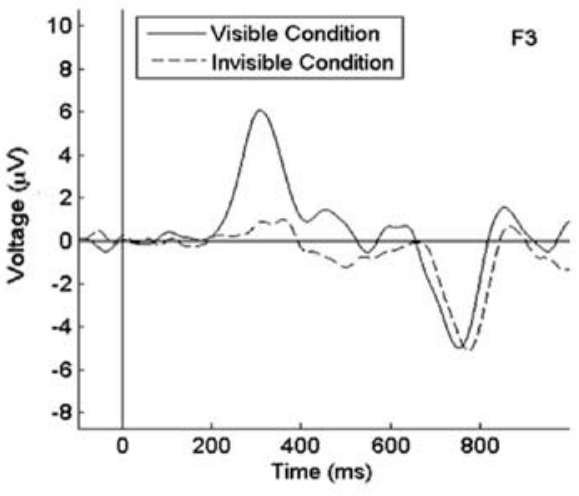

Figure 3. ERPs elicited by visible and invisible words. $\boldsymbol{a}$, Time course of responses to visible and invisible words at different electrodes. The $x$-axis shows time, and the $y$-axis shows electrodes; the color scale is expressed in microvolts. Zero represents the first mask onset. Vertical lines indicate sample-word and test-word presentation. Small lines at the top of the graph code for the two time windows corresponding to the voltage scalp maps in $\boldsymbol{b}$. $\boldsymbol{b}$, Voltage scalp map for two windows indicated for visible and invisible conditions. The first difference started at $130 \mathrm{~ms}$ after sample-word presentation, as a P300a-like component. Then, a P1-like component was observed $\sim 200 \mathrm{~ms}$ after test-word presentation, for both conditions. c, Time course of the signal recorded from left frontal electrode F3.

interval 10-40 ms after test-word presentation (visible vs invisible: $F_{(1,14)}=11.803$; $p=0.005)$. Interestingly, in this case the power of oscillations was also enhanced and in exactly the same frequency range (visible vs invisible: $F_{(1,14)}=8.006 ; p=$ 0.015). Because of their short latency, these effects might reflect anticipatory processes that occur only when the subjects have seen the sample word (Courtemanche and Lamarre, 2005). This anticipation was possible because the interval between sample- and test-word presentation was fixed. The significantly shorter reaction times observed in the visible condition support this interpretation.

Finally, there were two significant effects related to test-word presentation that did not differentiate between visible and invisible conditions. First, there was a significant increase in phase synchrony in the frequency band from 63 to $83 \mathrm{~Hz}$ in the interval $185-240 \mathrm{~ms}$ after test-word presentation (visible vs baseline: $F_{(1,14)}=$ 22.869, $p<0.001$; invisible vs baseline: $F_{(1,14)}=18.720, p=0.001$; visible vs invis-
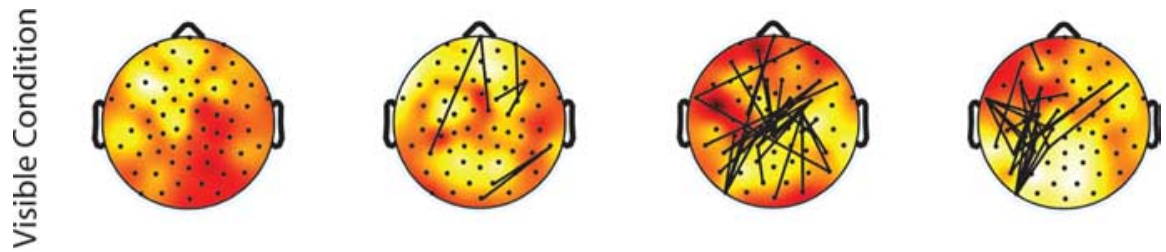

$-263:-111 \mathrm{~ms}$

$-111: 40 \mathrm{~ms}$

$40: 182 \mathrm{~ms}$

$182: 333 \mathrm{~ms}$
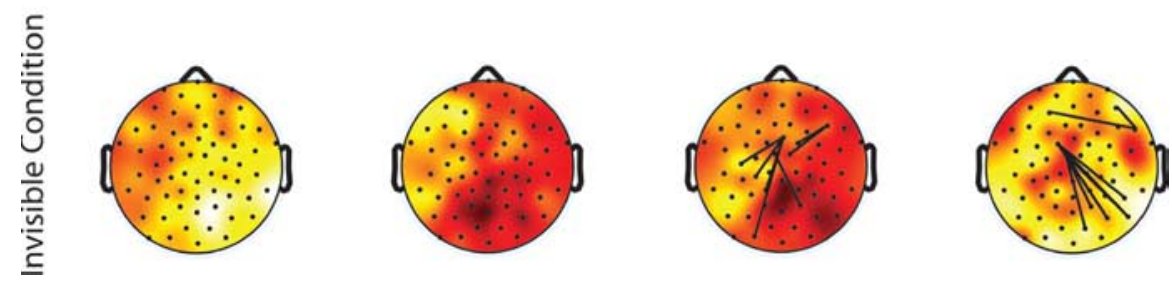

Figure 4. Scalp topography of induced gamma power and phase synchrony for the visible and invisible condition. Top row, Visible condition. Bottom row, Invisible condition. The background color indicates induced gamma power averaged in a $50-57 \mathrm{~Hz}$ frequency range. Each head represents the average of a $150 \mathrm{~ms}$ time window. Time 0 indicates the onset of the sample word. Lines connect pairs of electrodes displaying significant synchronization ( $p<0.000001)$. Gamma activity does not statistically differ between visible and invisible conditions. In contrast, phase synchrony is stronger in the visible condition during the $40-180 \mathrm{~ms}$ time window involving occipito, parieto, and frontal electrodes, with intrahemispheric and interhemispheric connections. In the window between 180 and $330 \mathrm{~ms}$, the pattern of phase synchrony lateralizes over the left hemisphere and restricts to occipitoparietal electrodes.

ible conditions: $F_{(1,14)}=0.199, p=0.663$ )

Second, there was a significant increase in power of gamma oscillations in a broad frequency range $(36-85 \mathrm{~Hz})$ during the interval of $155-230 \mathrm{~ms}$ after test-word presentation (visible vs baseline: $F_{(1,14)}=63.524, p<0.001$; invisible vs baseline: $F_{(1,14)}=52.626, p<0.001$; visible vs invisible condition: $F_{(1,14)}=$ $2.030, p=0.180)$.

In the ERP, test-word presentation evoked P1-N1 components, the latency, amplitude, and spatial distribution of which were similar in the visible and invisible conditions $\left(F_{(1,14)}=\right.$ 2.896; $p=0.115 ; 1.205 \mu \mathrm{V}$ difference).
Because word visibility was manipulated by changing mask luminance, it could be argued that the differences between visible and invisible conditions are attributable to luminance variations. To control for this possibility, we performed an additional analysis in which visible trials were subtracted from invisible trials (visible plus light effect) and contrasted with the difference between control visible and control invisible trials (referred to as light effect). This contrast should remove any remaining luminance effects, and persisting effects should only reflect processes related to conscious perception of the sample word. This analysis 
confirmed the results described above. There were two episodes of enhanced phase synchrony. One was associated with sampleword presentation (starting $80 \mathrm{~ms}$ after sample-word presentation; frequency range, $\left.50-57 \mathrm{~Hz} ; F_{(1,14)}=5.806 ; p=0.033\right)$ and the other with test-word presentation (starting $10 \mathrm{~ms}$ after testword presentation, frequency range $75-80 \mathrm{~Hz}\left(F_{(1,14)}=8.811\right.$, $p=0.012)$. In addition, the power of gamma oscillations was enhanced in association with the presentation of the test word (starting $20 \mathrm{~ms}$ after test-word presentation; frequency range, $60-75 \mathrm{~Hz} ; F_{(1,14)}=6.875 ; p=0.022$ ) (see supplemental Fig. 2, available at www.jneurosci.org as supplemental material).

Enhanced synchronization can be mimicked by volume conduction if it occurs with zero phase lag. In this case, the possibility needs to be considered that a single generator oscillating in the respective frequency increases its power leading to stronger synchronization of the signals measured on the scalp. Conversely, reduction in the activity of other oscillators can unmask the contribution of a single source, producing again stronger synchronization on the scalp. We consider it unlikely that this had occurred in the present experiment for the following reasons. First, if the observed synchronization were attributable to activation or inactivation of one or several generators, it should show up as a difference in the induced gamma activity or in the ERPs between experimental conditions. This was not the case in our experiment. During the interval of enhanced phase-locking, neither the ERPs nor the induced gamma oscillations differed between conditions, suggesting that the activity of the contributing generators was unchanged.

Second, synchronization caused by volume conduction must occur with zero phase difference between electrodes. In our experiment, the phase angles at which synchronization occurred were not centered on zero and exhibited considerable scatter ( $\pm \pi$ rads) (see supplemental Fig. 3, available at www.jneurosci. org as supplemental material). This means that the most prominent synchronization did not occur with zero phase lag, as expected from volume conduction. Furthermore, the scatter in phase lag implies that the observed synchronization occurs with varying time delays from trial to trial, which is incompatible with instantaneous volume conduction. Third, phase synchronization caused by volume conduction should exhibit a distancedependent gradient on the scalp (i.e., phase synchrony should decay with interelectrode distance). In our experiment there, was no evidence for a distance-dependent gradient in PLVs. In fact, the scalp topography shows that synchronization occurred more frequently between distant electrodes.

\section{Discussion}

The first electrographic difference between conscious and nonconscious stimulus processing was increased phase-locking of induced gamma oscillations across widely distributed cortical regions. This suggests that early large-scale synchronization could be the event that triggers ignition of the global workspace of consciousness, as postulated by Dehaene and Naccache (2001) and Dehaene et al. (2006).

\section{Local and long-range neural synchrony and their putative role in conscious and unconscious perception}

Our results show similar activation patterns at individual electrodes in the visible and invisible conditions, suggesting that the same neural generators are activated in both cases. In contrast, phase synchronization across electrodes clearly differentiated between conditions, suggesting enhanced long-range coordination of oscillatory activity only in the visible condition. Several au- thors have proposed that conscious perception should be related to coordinated dynamical states of the cortical network, rather than to the activation of specific brain regions (Fries et al., 1997, 2002; Engel et al., 1999; Engel and Singer, 2001; Thompson and Varela, 2001; Singer, 2002; Lamme, 2006). Our results offer direct support for this notion. In addition, our results and the results from a previous study investigating the neural dynamics of perceived and unperceived somatosensory stimuli (Palva et al., 2005) are in line with a recent proposal (Dehaene et al., 2006) relating unconscious processing of information with local coordination of neural activity in resonant loops of medium range and relating conscious perception with global coordination of distant neural activity by long-range synchronization. Interestingly, the global long-distance synchronization found in the visible condition was very transient and the earliest event differentiating conscious from nonconscious processing. After this, other electrophysiological measures, such as $\mathrm{P} 3 \mathrm{a}$ and theta oscillations, continue to differentiate between consciously and nonconsciously perceived words. This suggests that long-distance synchronization plays a role in triggering the cognitive processes associated with conscious awareness (Dehaene et al., 2006). However, it remains to be clarified through which mechanism long-distance synchronization exerts an impact on subsequent cognitive processes.

The transient character of the long-distance synchronization is not entirely compatible with models such as global workspace (Dehaene and Changeux, 2005; Dehaene et al., 2006) and reentrant activity (Lamme, 2006) because these predict a more sustained response for consciously perceived stimuli. Our results show increased neural synchrony for the visible condition, which lasts $\sim 100 \mathrm{~ms}$ but reaches significance only during a short time window $(\sim 50 \mathrm{~ms})$, suggesting that neural synchronization could last longer but is nonetheless transient.

The discrepancy between sustained and transient activity found in different studies could also be attributable to the different experimental paradigms. Most of the experiments that have reported sustained activity used either the attentional blink paradigm (Gross et al., 2004; Sergent et al., 2005) or inattentional blindness (Dehaene and Changeux, 2005). It is still controversial whether the attentional blink paradigm assesses conscious perception or memory processes. It has been argued that subjects could have conscious access to the stimulus at the moment of its presentation, yet simply forget it when they are asked to report it (Wolfe, 1999). In fact, experiments on the related phenomenon of change blindness show that unattended objects could be fleetingly represented and reported but that their representations disappear as soon as a new object is presented or if the eyes move to a new location (Landmann et al., 2003). Thus, the sustained activity often reported using such experimental paradigms could reflect the transfer or maintenance of a stable representation in working memory and are not necessarily a correlate of conscious perception itself.

\section{Early wave of activation that distinguishes conscious from unconscious processing}

In contrast to previous experiments, the results of which suggest a late wave of activation as correlate of sensory awareness (Gross et al., 2004; Sergent et al., 2005), our results indicate that access to consciousness is triggered by an early coordination (synchronization) of widely distributed neuronal assemblies starting as early as $80 \mathrm{~ms}$ after stimulus presentation [see also Palva et al. (2005) and Fries et al. (2001) for a similar finding]. This difference might be explained by two factors. First, the fast stream of stimuli used 
in our experiment might have imposed pressure on the perceptual processes, leading to a shortening of the processing time devoted to each stimulus. Second, in contrast to previous experiments, in our study the neural processes related to perception were segregated in time from those related to decision making and execution of motor responses. The late correlates of conscious perception described previously might thus reflect a mixture of cognitive processes (e.g., perception and decision making) that may have masked the brief and early episode of phaselocking. The responses observed after test-word presentation support this latter interpretation. The presentation of the test word also triggered a sequence of cognitive processes, but these could be executed without imposed delays (e.g., perception, decision making, and acting). In this case, the induced synchronization occurred with much longer latency ( $\sim 200 \mathrm{~ms})$ and was in the range described in previous experiments. The present finding that the first electrographic signature of conscious processing has a short latency is in agreement with the evidence that areas at the highest level of the processing hierarchy (frontal areas) become active as early as $100 \mathrm{~ms}$ after stimulation (Nowak and Bullier, 1997) and that high cognitive processes, such as stimulus categorization, can be accomplished within $120 \mathrm{~ms}$ (Thorpe et al., 1996; Kirchner and Thorpe, 2006). In addition, our results agree with a series of studies showing that conscious and unconscious perception can be rapidly distinguished after stimulus presentation (Dehaene et al., 2001; Pins and Ffytche, 2003; Palva et al. 2005). Additionally, in the same time window, where we found a synchronization episode (80-120 ms), Walsh and Cowey (1998) found that applying a TMS magnetic pulse over V1 and/or circumstriate areas can impair visual perception of a briefly presented stimulus, suggesting that this time window is relevant for conscious perception. In accordance with the present data, these results suggest that the brain can process information remarkably fast and that the divergence of conscious and unconscious processing occurs within $100 \mathrm{~ms}$ after stimulus presentation, implying that this early wave of activation might be an essential correlate of conscious perception (Pins and Ffytche, 2003).

\section{Late correlates of conscious processing}

Previous studies investigating electrographic correlates of conscious and unconscious processing have evaluated either stimulus-locked ERPs (Sergent et al., 2005) or dynamic measures such as spectral power or phase synchrony (Srinivasan et al., 1999; Gross et al., 2004; Doesburg et al., 2005; Palva et al., 2005). The combination of the different methods applied here allows for a more detailed temporal characterization of the putative EEG events differentiating between conscious and unconscious processing. Together, the data suggest that only consciously perceived stimuli give rise to a cascade of processes that have distinct electrophysiological signatures. In summary, these consisted of (1) an early and global phase-locking of gamma oscillations, (2) an enhancement of a P3a-like component of the ERP and of sustained theta oscillations over frontal areas that are likely to reflect transfer and maintenance of the perceived stimuli in working memory, and (3) an enhancement of power and phaselocking of gamma oscillations before test stimulus presentation that may be a correlate of the anticipation of the matching between short-term memory contents and sensory input. Our results on ERPs agree with the data of Sergent et al. (2005), which suggests that ERPs evoked by perceived and unperceived stimuli start to diverge around $270 \mathrm{~ms}$. Interestingly, these ERP differences occur only after the end of the transient increase in phase synchrony. Thus, it seems as if the fast and transient large-scale synchronization enhances the saliency of the activation patterns sufficiently to permit access to consciousness and thereby triggers a sequence of processes such as perception stabilization, maintenance in working memory, and generation of expectancies that are associated with conscious awareness. It remains to be clarified whether the early large-scale synchronization is already the neuronal correlate of phenomenal awareness or whether awareness emerges only from the entirety of the processes following this coordinated state.

\section{Long-range synchronization, conscious perception, and the depth of processing}

It can be argued that the electrophysiological signatures associated with conscious perception are simply a reflection of more extensive processing rather than reflecting mechanisms specifically associated with awareness. We consider this as unlikely for several reasons. The finding that visible and invisible words induced gamma oscillations of similar power and distribution suggests that invisible words were thoroughly processed. In addition, in the control experiment with the subliminal priming task, we evaluated the depth of processing of the unperceived word using the same protocol as in the main experiment. Prime words, although not perceived, had a clear behavioral effect indicating that the unconsciously perceived words are processed. Therefore, we consider it likely that the key event mediating access to consciousness is the early long-distance synchronization of neural assemblies, rather than the mere depth of processing in the various cortical areas involved in written word processing.

\section{References}

Bar M, Tootell R, Schacter D, Greve B, Fischl B, Mendola J, Rosen B, Dale A (2001) Cortical mechanism specific to explicit visual object recognition. Neuron 29:529-535.

Beck D, Rees G, Frith C, Lavie N (2001) Neural correlates of change detection and change blindness. Nat Neurosci 4:645-650.

Burgess A, Gruzelier J (1999) Methodological advances in the analysis of event-related desynchronization data: reliability and robust analysis. In: Handbook of electroencephalography and clinical neurophysiology, revised series, Vol 6 (Pfurtscheller G, Lopes da Silva FH, eds), pp 139-158. Amsterdam: Elsevier Science.

Cappelletti M, Cipolotti L (2006) Unconscious processing of Arabic numerals in unilateral neglect. Neuropsychologia 44:1999-2006.

Carmel D, Lavie N, Rees G (2005) Conscious awareness of flicker in human involves frontal and parietal cortex. Curr Biol 16:907-911.

Courtemanche R, Lamarre Y (2005) Local field potential oscillations in primate cerebellar cortex: synchronization with cerebral cortex during active and passive expectancy. J Neurophysiol 93:2039-2052.

Dehaene S, Changeux J (2005) Ongoing spontaneous activity controls access to consciousness: a neuronal model for inattentional blindness. PLoS Biol 3:911-927.

Dehaene S, Naccache L (2001) Towards a cognitive neuroscience of consciousness: basic evidence and a workspace framework. Cognition 79:1-38.

Dehaene S, Naccache L, Le Clec HG, Koechlin E, Mueller M, DehaeneLambertz G, van de Moortele PF, Le Bihan D (1998) Imaging unconscious semantic priming. Nature 395:597-600.

Dehaene S, Naccache L, Cohen L, Le Bihan D, Mangin JF, Poline JB, Riviere D (2001) Cerebral mechanisms of word masking and unconscious repetition priming. Nat Neurosci 4:752-758

Dehaene S, Jobert A, Naccache L, Ciuciu P, Poline JB, Le Bihan D, Cohen L (2004) Letter binding and invariant recognition of masked words: behavioral and neuroimaging evidence. Psychol Sci 15:307-313.

Dehaene S, Changeux JP, Naccache L, Sackur J, Sergent C (2006) Conscious, preconscious, and subliminal processing: a testable taxonomy. Trends Cogn Sci 10:204-211.

Delorme A, Makeig S (2004) EEGLAB: an open source toolbox for analysis of single-trial EEG dynamics including independent component analysis. J Neurosci Methods 134:9-21. 
Doesburg S, Kitajo K, Ward L (2005) Increased gamma-band synchrony precedes switching of conscious perceptual objects in binocular rivalry. NeuroReport 16:1139-1142.

Donchin E (1981) Surprise!. . Surprise? Psychophysiology 18:493-513.

Engel A, Singer W (2001) Temporal binding and the neural correlates of sensory awareness. Trends Cogn Sci 5:16-25.

Engel A, Fries P, Konig P, Brecht M, Singer W (1999) Temporal binding, binocular rivalry, and consciousness. Conscious Cogn 8:128-151.

Fries P, Roelfsema P, Engel A, Konig P, Singer W (1997) Synchronization of oscillatory responses in visual cortex correlates with perception in interocular rivalry. Proc Natl Acad Sci USA 94:12699-12704.

Fries P, Reynolds JH, Rorie AE, Desimone R (2001) Modulation of oscillatory neuronal synchronization by selective visual attention. Science 291:1560-1563.

Fries P, Schroeder J, Roelfsema P, Singer W, Engel A (2002) Oscillatory neuronal synchronization in primary visual cortex as a correlate of stimulus selection. J Neurosci 22:3739-3754.

Goebel R, Muckli L, Zanella FE, Singer W, Stoerig P (2001) Sustained extrastriate cortical activation without visual awareness revealed by fMRI studies of hemianopic patients. Vision Res 41:1459-1474.

Grill-Spector K, Kushnir T, Hendler T, Malach R (2000) The dynamics of object-selective activation correlate with recognition performance in humans. Nat Neurosci 1:91-100.

Gross J, Schmitz I, Schnitzler K, Kessler K, Shapiro B, Hommel A, Schnitzler A (2004) Modulation of long-range neural synchrony reflects temporal limitations of visual attention in humans. Proc Natl Acad Sci USA 101:13050-13055.

Jensen O, Tesche C (2002) Frontal theta activity in humans increases with memory load in a working memory task. Eur J Neurosci 15:1395-1399.

Kirchner H, Thorpe S (2006) Ultra-rapid object detection with saccadic eye movements: visual processing speed revisited. Vision Res 46:1762-1776.

Kleinschmidt A, Buchel C, Hutton C, Friston C, Frackowiak R (2002) The neural structure expressing perceptual hysteresis in visual letter recognition. Neuron 34:659-666.

Lachaux J, Rodriguez E, Martinerie J, Varela F (1999) Measuring phasesynchrony in brain signals. Hum Brain Mapp 8:194-208.

Lamme V (2006) Towards a true neural stance on consciousness. Trends Cogn Sci 10:494-501.

Landmann R, Spekreijse H, Lamme V (2003) Large capacity storage of integrated objects before change blindness. Vision Res 43:149-164.

Marcel A (1983) Conscious and unconscious perception: experiments on visual masking and word recognition. Cogn Psychol 15:197-237.

Marois R, Yi D, Chun M (2004) The neural fate of consciously perceived and missed events in the attentional blink. Neuron 41:465-472.

Meador K, Ray P, Echauz J, Loring D, Vachtsevanos G (2002) Gamma coherence and conscious perception. Neurology 59:847-854.

Moutoussis K, Zeki S (2002) The relationship between cortical activation and perception investigated with invisible stimuli. Proc Natl Acad Sci USA 99:9527-9532.
Moutoussis K, Zeki S (2006) Seeing invisible motion: a human fMRI study. Curr Biol 16:574-579.

Naccache L, Gaillard R, Adam C, Hasboun D, Clemenceau S, Baulac M, Dehaene S, Cohen L (2005) A direct intracranial record of emotions evoked by subliminal words. Proc Natl Acad Sci USA 102:7713-7717.

Nakatani C, Ito J, Nikolaev A, Gong P, van Leeuwen C (2005) Phase synchronization analysis of EEG during attentional blink. J Cog Neurosci 17:1969-1979.

Nowak LG, Bullier J (1997) The timing of information transfer in the visual system. In: "Extrastriate cortex" cerebral cortex, Vol 12 (Kass J, Rockland K, Peters A, eds), pp 205-241. New York: Plenum.

Palva S, Linkenkaer-Hansen K, Naatanen R, Palva M (2005) Early neural correlates of conscious somatosensory perception. J Neurosci 25:5248-5258.

Pins D, Ffytche D (2003) The neural correlates of conscious vision. Cereb Cortex 13:461-474.

Renault B, Signoret JL, Debruille B, Breton F, Bolgert F (1989) Brain potentials reveal covert facial recognition in prosopagnosia. Neuropsychologia 27:905-912.

Rodriguez E, Lachaux JP, Martinerie J, Renault B, Varela FJ (1999) Perception's shadow: long-distance synchronization of human brain activity. Nature 397:430-433.

Schack B, Klimesch W, Sauseng P (2005) Phase synchronization between theta and upper alpha oscillations in a working memory task. Int J Psychophysiol 57:105-114.

Sergent C, Baillet S, Dehaene S (2005) Timing of the brain events underlying access to consciousness during the attentional blink. Nat Neurosci 8:1391-1400.

Singer W (2002) Consciousness from neurobiological perspective. In: Neural correlates of consciousness (Metzinger T, ed), pp 121-137. Cambridge, MA: MIT.

Srinivasan R, Russell D, Edelman G, Tononi G (1999) Increased synchronization of neuromagnetic responses during conscious perception. J Neurosci 19:5435-5448.

Thompson E, Varela F (2001) Radical embodiment: neural dynamics and consciousness. Trends Cogn Sci 10:418-425.

Thorpe S, Fize D, Marlot C (1996) Speed of processing in the human visual system. Nature 381:520-522.

Varela F, Lachaux J, Rodriguez E, Martinerie J (2001) The brain web: phase synchronization and large-scale integration. Nat Rev Neurosci 2:229-239.

Vuilleumier P, Armony J, Clarke K, Husain M, Driver J, Dolan R (2002) Neural response to emotional faces with and without awareness: eventrelated fMRI in a parietal patient with visual extinction and spatial neglect. Neuropsychologia 40:2156-2166.

Walsh V, Cowey A (1998) Magnetic stimulation studies of visual cognition. Trends Cogn Sci 2:103-110.

Wolfe JM (1999) Inattentional amnesia. In: Fleeting memories: cognition of brief visual stimuli (Coltheart V, ed), pp 71-91. Cambridge, MA: MIT. 